\title{
COVID-19 en pacientes en hemodiálisis crónica. Experiencia clínica y resultados en la Ciudad de México
}

\author{
COVID-19 in patients with chronic hemodialysis. Clinical \\ experience and results in Mexico City
}

\author{
JM Ramos Gordillo, ${ }^{*}$ C Méndez Valdez, ${ }^{\ddagger}$ R Patiño Ortega, $\$$ \\ LG Hernández Vázquez, `D Dávila Palomeque," MV Magallanes Mendoza,** \\ PC Ruiz Palacios, ${ }^{\neq \neq}$A Rogel Millán, $\$ \S$ José Carlos Peña Rodríguez ${ }^{\text {qा }}$
}

Citar como: Ramos GJM, Méndez VC, Patiño OR, Hernández VLG, Dávila PD, Magallanes MMV et al. COVID-19 en pacientes en hemodiálisis crónica. Experiencia clínica y resultados en la Ciudad de México. Acta Med Grupo Angeles. 2021; 19 (2): 221-228. https://dx.doi.org/10.35366/100446

\section{Resumen}

Introducción: Es un informe multicéntrico de los casos de COVID-19 en pacientes bajo hemodiálisis crónica (HC). Material y métodos: Estudio observacional, retrospectivo, en 3,723 pacientes en hemodiálisis crónica. Se incluyeron a aquellos diagnosticados con COVID-19 entre el primero de abril y el 15 de julio del 2020. Se clasificaron en casos con sospecha y confirmados. Resultados: Se identificaron 373 sucesos dudosos $(10 \%)$ en hemodiálisis. En $160(40 \%)$ se ratificó el diagnóstico de COVID-19, con una incidencia del 4.3\%; 94 hombres y 66 mujeres. Promedio de edad de $55 \pm 15$ años (20-88 años). De esos enfermos 73 fallecieron, con una tasa de letalidad de $45 \%$ (73/160). La etiología más frecuente fue la nefropatía diabética en 64 casos (40\%), seguida por causa no determinada en 46 (28\%), nefroangioesclerosis en 31 (19\%). Los síntomas más comunes fueron: fiebre, tos, disnea y diarrea. El promedio de la saturación de oxígeno en los pacientes sospechosos fue: 81 $\pm 7 \%$. Los casos confirmados cursaron con linfopenia que fue mayor en los contagiados que fallecieron. Conclusión: La tasa de letalidad fue muy elevada (45\%). Los sujetos a hemodiálisis son un grupo de elevada susceptibilidad al SARS-CoV-2 y con riesgo alto de fallecer con síndrome respiratorio agudo, cuando se les compara con la población general.

Palabras clave: COVID-19, hemodiálisis crónica, letalidad, incidencia.

\section{Abstract}

Introduction: This is a report of COVID-19 in chronic hemodialysis patients. Material and methods: This is a retrospective and observational study in chronic hemodialysis patients. All patients diagnosed with COVID-19 from April 1st to July 15th 2020 were included. The diagnosis was established with real-time polymerase chain reaction (RT-PCR) from throat and nose swabbing, in all suspected cases. Results: 373 suspected cases were identified (10\%), 160 (40\%) were confirmed cases. With an incidence of $4.3 \%$; 66 females and 94 males: average age $55 \pm 15$ years ( $20-88$ years), 73 people died $(45 \%)$. The ratified diagnosis of all such cases were: Diabetic nephropathy 64 cases $(40 \%)$, unknown cause 46 cases (28\%), nephrosclerosis $31(19 \%)$ and other causes $12 \%$. The most common symptoms were: fever, cough, dyspnea and diarrhea. The average value of oxygen saturation in suspected cases was $81 \pm 7 \%$. Lymphocyte count was lower in patients who died. Conclusion: The percent mortality in this group of patients in maintenance hemodialysis was very high (45\%) in comparison with the general population (5-10\%). Of these, $40 \%$ were diabetics. All patients that developed acute respiratory syndrome died.

Keywords: COVID-19, chronic hemodialysis, lethality, incidence.

\section{Centro de Diagnóstico Ángeles (CEDIASA). México.}

* Subdirector Médico.

Director de la Unidad de Hemodiálisis Acoxpa.

§ Director de la Unidad de Hemodiálisis Guadalupe.

" Director de la Unidad de Hemodiálisis Iztapalapa.

II Director de la Unidad de Hemodiálisis Mocel.

** Director de la Unidad de Hemodiálisis Polanco.

‡¥ Director de la Unidad de Hemodiálisis Revolución.

$\$ \S$ Director de la Unidad de Hemodiálisis Toluca.

ฯศ Director Médico.
Correspondencia:

José Carlos Peña Rodríguez

Correo electrónico: josecarlos.pena@saludangeles.com

Aceptado: 17-11-2020.

www.medigraphic.com/actamedica

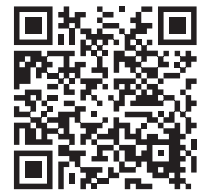




\section{INTRODUCCIÓN}

En diciembre de 2019, en Wuhan, China, se informaron las primeras ocurrencias de una neumonía de causa desconocida, pero con características clínicas muy similares a una de etiología viral. ${ }^{1}$ Posteriormente, la secuenciación de genes de este patógeno confirmó que el agente etiológico infectante era un nuevo coronavirus, el SARS-CoV-2. ${ }^{2}$

La infección por SARS-CoV-2 mostró desde su inicio su alta capacidad de transmisión en la comunidad, y en la actualidad ya afecta a más de 200 países alrededor del mundo. La Organización Mundial de la Salud catalogó a esta enfermedad como una pandemia de preocupación internacional. ${ }^{3}$

La presentación clínica es muy variable, ya que en un $80 \%$ de las ocasiones la evolución es asintomática o con sintomatología leve; un 15\% de los pacientes presentan neumonía unilateral o bilateral y el 5\% restante desarrolla neumonía bilateral con síndrome de distrés respiratorio, que amerita apoyo ventilatorio mecánico y atención médica en unidades de cuidados intensivos. En muchos casos graves, la respuesta inmune puede desencadenar una fuerte reacción inflamatoria, acompañada de una tormenta de citocinas que empeoran los síntomas respiratorios y pueden provocar la muerte. ${ }^{4}$ Los mecanismos fisiopatológicos implicados en la variabilidad del curso clínico de este mal no están aún esclarecidos. Informes de diferentes investigadores de todo el mundo han identificado enfermos con riesgo elevado de desarrollar complicación muy grave y muerte. ${ }^{4}$ Este grupo incluye sujetos mayores de 60 años, con padecimiento cardiaco o pulmonar subyacente, enfermedad renal crónica (ERC), obesidad, diabetes e hipertensión arterial. En investigaciones multicéntricas realizadas en febrero y marzo en más de 10,000 personas, principalmente de China, Italia y España, se estimó una tasa de mortalidad en la población en general entre 1.4 y $8 \%{ }^{5,6}$ En los casos de enfermos que ingresan a la Unidad de Cuidados Intensivos ( $\mathrm{UCI}$ ) la tasa de mortalidad oscila del 16 al 78\%. Estas tasas tienen una variación tan amplia porque se comportan de acuerdo con los criterios clínicos que se aplican para ingresar a los enfermos a la $\mathrm{UCl}^{7}$

La ERC de cualquier etiología y en cualquier estadio ha sido identificada como un factor de peligro de mortalidad. En un estudio realizado en China, se demostró que un incremento en la concentración de creatinina en suero se asoció con un riesgo relativo (RR) de muerte de aproximadamente cuatro veces. ${ }^{8}$

Los pacientes en hemodiálisis, desde el primer informe de Wuhan en China, se consideraron como sujetos de muy alto riesgo ya que presentaron una elevada mortalidad.

La razón de la clasificación de gravedad y mortalidad altas en los sometidos a hemodiálisis derivan de que, en su gran mayoría, son sujetos de edad avanzada y la ERC se asocia a un gran número de comorbilidades: diabetes mellitus, hipertensión arterial, cardiopatía isquémica, enfermedad pulmonar obstructiva crónica, desnutrición, insuficiencia arterial periférica, entre las principales. Además, los sujetos a hemodiálisis, debido a su trastorno de base, tienen una reducción en su respuesta inmunológica que los hace altamente vulnerables a este contagio. 9,10

En las unidades de hemodiálisis de prácticamente todo el mundo se han tomado medidas preventivas y de aislamiento, buscando evitar la dispersión del virus. Sin embargo, se desconocen las características específicas y el comportamiento de la enfermedad en esta población.

El objetivo de este estudio observacional fue describir la presentación, curso clínico, y factores de riesgo de estos pacientes en hemodiálisis crónica. Se describe además el primer caso de esa serie, que resultó sorpresivo, donde el enfermo falleció por su evolución tan violenta.

\section{MATERIAL Y MÉTODOS}

Es un análisis observacional, descriptivo, analítico y retrospectivo de pacientes con enfermedad renal crónica en estadio 5/5 que reciben hemodiálisis crónica en alguna de las siete unidades de hemodiálisis (UH) con que cuenta el Centro de Diagnóstico Ángeles (CEDIASA) en la Ciudad de México y el área metropolitana. Todos los afectados firmaron consentimiento informado y se les garantizó la protección de sus datos personales. La investigación incluyó a todos los receptores de hemodiálisis crónica, diagnosticados con COVID-19, entre el $1^{\circ}$ de abril y el 15 de julio de 2020. El diagnóstico se realizó mediante la prueba de reacción en cadena de la polimerasa transcriptasa reversa (RT-PCR) de muestras de secreciones faríngeas de la boca y nariz, a todos los enfermos con sospecha de infección por SARS-CoV-2. Ellos recibían hemodiálisis de alta eficiencia, tres veces a la semana, con máquinas marca Fresenius modelo 4008S con dializadores de polisulfona.

$\mathrm{Al}$ inicio de la pandemia se establecieron las siguientes medidas tratando de evitar la propagación del virus. Se colocaron uno o más "Módulos de Atención Respiratoria", a la entrada de las instalaciones de las siete $U H$, a fin de que todos los internados y su familiar acompañante fueran evaluados mediante un pequeño interrogatorio sobre la presencia de sintomatología sugestiva de infección por SARS-CoV-2, además de toma de temperatura corporal y determinación del porcentaje de saturación de oxígeno.

Se clasificaron tales pacientes en sospechosos y confirmados. A continuación, se especifican los criterios que se utilizaron en esta división.

Caso incierto. Persona de cualquier edad que en los últimos siete días haya presentado al menos dos de los 


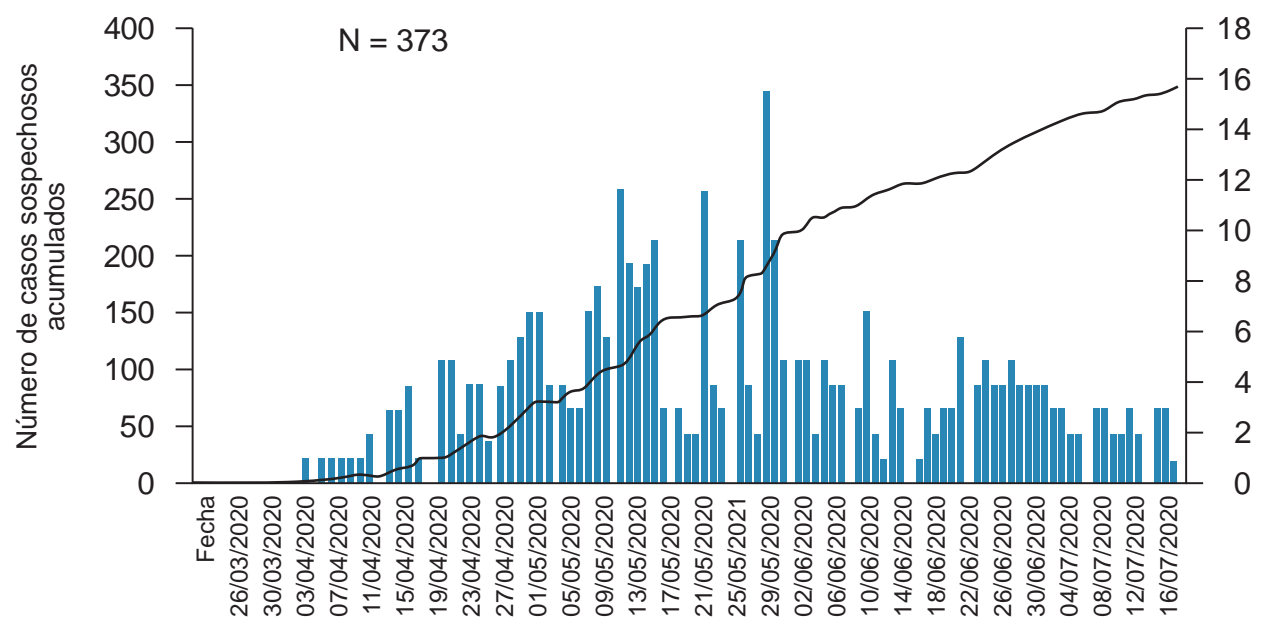

Figura 1:

Gráfica de casos sospechosos nuevos y acumulados.

siguientes signos y síntomas: tos, fiebre o cefalea, acompañados de al menos uno de los siguientes signos o síntomas: disnea, artralgias, mialgias, odinofagia, disgeusia, rinorrea, conjuntivitis o dolor torácico, así como reducción en la saturación de oxígeno.

Caso confirmado. Persona que cumpla con la definición operacional de caso sospechoso y que cuente con diagnóstico ratificado mediante la prueba de reacción en cadena de la polimerasa con transcriptasa inversa (RT-PCR), realizada por la Red Nacional de Laboratorios de Salud Pública, reconocidos por el Instituto Nacional de Referencia Epidemiológica (INDRE).

\section{Medidas de protección}

En todas las UH se dispusieron despachadores con solución alcoholada al $70 \%$ para que todas las personas que ingresaran pudieran realizar la desinfección de sus manos, a quienes se les orientó para evitar aglomeraciones.

Además, se vigiló el cumplimiento de las disposiciones de la "Jornada Nacional de Sana Distancia" y se reforzó la higiene de manos entre el personal, así como los internados y familiares que se encontraban dentro de las $U H$.

A aquellos pacientes que cumplieron con la definición operacional (caso dudoso o confirmado), se les proporcionó un tapabocas y se les realizó una valoración por parte del médico nefrólogo, y en caso de considerarlo necesario, se les envió a su servicio médico correspondiente para que se les realizara el protocolo diagnóstico de infección por SARS-CoV-2.

En todos los pacientes identificados como sospechosos o corroborados se obtuvieron los siguientes datos: edad, género, etiología de la ERC (nefropatía diabética, nefropatía hipertensiva, enfermedad poliquística renal, hipoplasia renal y nefropatía lúpica); índice de masa corporal, tiempo en hemodiálisis, presencia de las siguientes comorbilidades: diabetes, presión arterial alta, lupus, cardiopatía isquémica, entre otras. Se identificó la sintomatología más frecuente y se recabaron los resultados de laboratorio y gabinete siguientes: hemoglobina, leucocitos, plaquetas, índice de diálisis Kt/V, albúmina, proteínas totales, grupo sanguíneo, radiografía y/o tomografía axial computarizada (TAC) de tórax, finalmente se analizó la mortalidad.

Análisis estadístico. Las variables cualitativas fueron analizadas y presentadas mediante la determinación de frecuencias absolutas y relativas. Las variables cuantitativas continuas quedaron expresadas como promedio y desviación estándar. La asociación de variables cualitativas fue evaluada con la prueba $\chi^{2}$. El análisis unidireccional de la prueba de t de Student se utilizó para comparar las variables continuas de los conjuntos. Todo el análisis estadístico fue realizado con el software SPSS versión 22. La significancia estadística fue considerada con un valor de $\mathrm{p}<0.05$.

\section{RESULTADOS}

De un total de 3,723 pacientes que acuden regularmente a recibir sesión de hemodiálisis en alguna de las siete unidades con que cuenta el Centro de Diagnóstico Ángeles (CEDIASA) se identificaron un total de $373(10 \%)$ casos sospechosos (Figura 1). Se incluyeron 160 personas a quienes se les diagnosticó COVID-19, 94 (59\%) eran de sexo masculino y 66 (41\%) del femenino. El promedio de edad fue de $55 \pm 15$ años (20-88 años). La tasa de incidencia fue de $4.3 \%$ (Figura 2). Al final del periodo se identificaron 73 pacientes que habían fallecido, con una tasa de letalidad de $45 \%$ (Tabla 1). 
El promedio de índice de masa corporal fue de $26 \pm$ 5 , el tiempo promedio en hemodiálisis de los pacientes es $49 \pm 38$ meses. La etiología más frecuente fue la nefropatía diabética con $64(40 \%)$, seguida por la etiología no determinada con 46 (29\%) y la nefroangioesclerosis con 31 (20\%), entre las principales (Tabla 2). Los síntomas más comunes de COVID-19 resultaron ser: disnea $(65 \%)$, tos $(60 \%)$, fiebre $(50 \%)$, mialgias $(46 \%)$, diarrea $(35 \%)$, cefalea (25\%), dolor torácico (14\%) y odinofagia (14\%). El promedio de la saturación de oxígeno en los internados sospechosos fue: $81 \pm 7 \%$.

De acuerdo con la evolución clínica, la población del estudio fue dividida en dos conjuntos: recuperados y fallecidos, los cuales fueron comparados. En cuanto a la edad, género, etiología, índice de masa corporal, tiempo en hemodiálisis, grupo sanguíneo, concentraciones de hemoglobina y albúmina, no existieron diferencias entre ambos conjuntos. El número de linfocitos fue mayor en el grupo de pacientes recuperados comparado con el de fallecidos (1,142 \pm 396 versus $978 \pm 330, p<0.005)$. En el grupo de pacientes fallecidos, el porcentaje de sobrepeso fue mayor que en la serie de enfermos recuperados $(73 \%$ versus 55\%, p < 0.22) (Tabla 2).

Los factores de riesgo para fallecer por COVID-19 en la cohorte estudiada fueron: la presencia de sobrepeso u obesidad y un recuento total de linfocitos menor a 1,000/ $\mathrm{mL}$ (Tabla 3).

Primer caso estudiado que falleció el $\mathbf{1 0}$ de abril del 2020. Se trató de una paciente de 59 años del sexo femenino, quien ingresó el viernes 10 de abril al turno de las 10 horas, asintomática, afebril y con $88 \%$ de saturación de oxígeno.
A las dos horas de su admisión presentó malestar orofaríngeo, disnea, visión borrosa y cefalea, en ese momento la saturación de $\mathrm{O}_{2}$ era de $56 \%$. Se inició oxigenación con puntas nasales, se detectó una desaturación progresiva de dicho gas, que evolucionó a pérdida de conciencia, disociación toracoabdominal, de tal modo que hubo necesidad de intubarla. En la siguiente hora, la paciente ya intubada fue trasladada al hospital, donde ingresó grave y a las dos horas falleció.

Los días previos, la paciente había cursado asintomática y en ningún momento acusó sintomatología sospechosa, hasta la iniciación de la disnea y la desaturación de oxígeno a las dos horas de iniciada la hemodiálisis y cuatro horas antes de su fallecimiento.

Este evento sorprendió por la violencia de su evolución y es un buen ejemplo del comportamiento que pueden tener dichos pacientes en hemodiálisis con severo daño renal y neumonía asintomática, más comorbilidades como diabetes mellitus, obesidad e hipertensión arterial, en una mujer relativamente joven. Se había mantenido en hemodiálisis $(\mathrm{Hd})$ en esta unidad en los últimos cinco años.

A raíz de este caso y de su evolución tan grave se tomaron medidas más drásticas y mayores cuidados, las que se han mantenido hasta la fecha de este informe.

\section{DISCUSIÓN}

La infección por el virus SARS-CoV-2 es una pandemia que representa un gran desafío para los servicios de salud de todo el mundo. Diversos artículos han informado que la mortalidad en la población en general por COVID-19 se ve incrementada por algunas comorbilidades, como enfermedad cardiovascular, diabetes mellitus, enfermedad

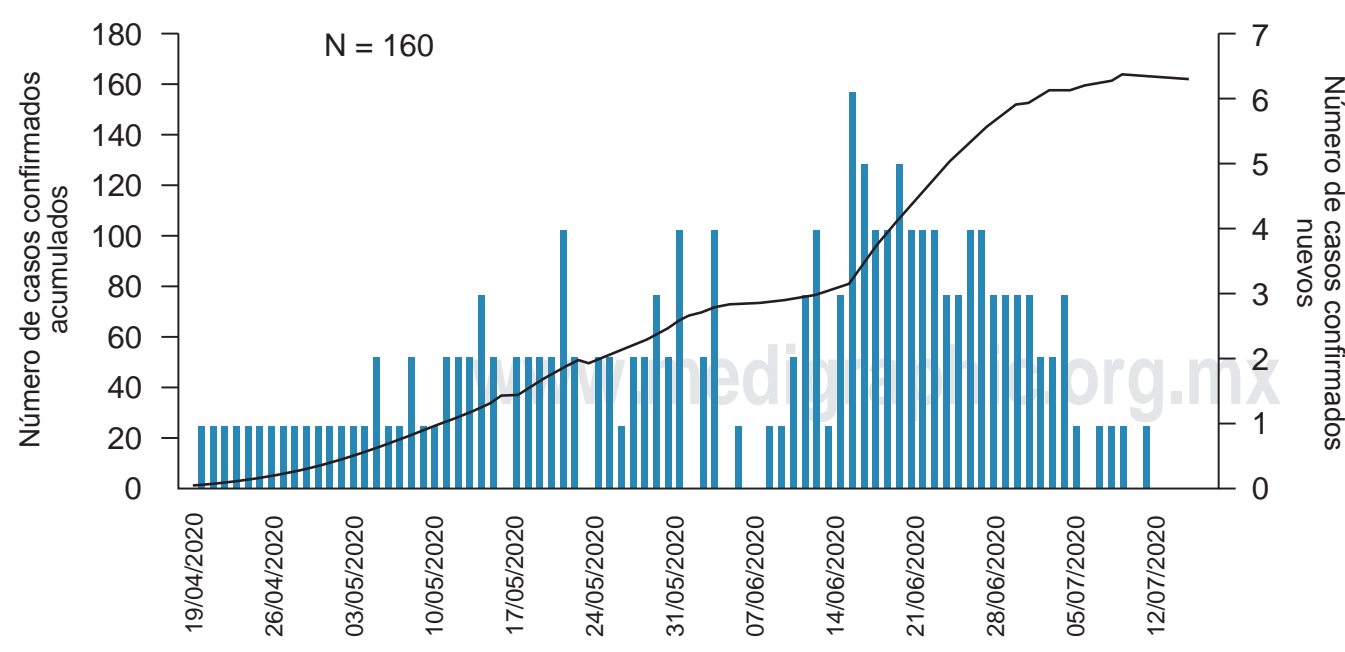

Figura 2:

Gráfica de casos confirmados nuevos y acumulados.

Casos confirmados nuevos 
Tabla 1: Distribución de casos sospechosos, confirmados, pacientes recuperados y decesos por unidad de hemodiálisis.

\begin{tabular}{|c|c|c|c|c|c|}
\hline Unidad de Hemodiálisis & $\begin{array}{c}\text { Pacientes } \\
n\end{array}$ & $\begin{array}{c}\text { Pacientes } \\
\text { sospechosos } \\
n\end{array}$ & $\begin{array}{c}\text { Pacientes con } \\
\text { COVID-19 (+) } \\
n\end{array}$ & $\begin{array}{c}\text { Pacientes } \\
\text { recuperados } \\
n\end{array}$ & $\begin{array}{c}\text { Decesos } \\
\text { COVID-19 } \\
n\end{array}$ \\
\hline Polanco & 1,201 & 98 & 61 & 34 & 27 \\
\hline Guadalupe & 718 & 51 & 18 & 5 & 13 \\
\hline Revolución & 346 & 57 & 10 & 7 & 3 \\
\hline Acoxpa & 209 & 21 & 7 & 3 & 4 \\
\hline Iztapalapa & 671 & 77 & 24 & 16 & 8 \\
\hline Toluca & 446 & 45 & 30 & 16 & 14 \\
\hline Mocel & 132 & 24 & 10 & 6 & 4 \\
\hline Total & 3,723 & 373 & 160 & 87 & 73 \\
\hline
\end{tabular}

pulmonar obstructiva crónica, presión alta y cáncer. ${ }^{1,2}$ Sin embargo, el pronóstico de los pacientes con COVID-19 en hemodiálisis aún no está claro y se necesitan más datos para conocer el comportamiento clínico de este padecimiento.

Desde la aparición de los primeros eventos de COVID-19 en este país, se implementaron las siguientes estrategias en todas las unidades de hemodiálisis del consorcio para evitar brotes de contagios: instalación de módulos de evaluación respiratoria "triaje respiratorio" en la entrada, donde se interrogaba a cada paciente sobre sintomatología sugestiva: fiebre, tos, disnea, cefalea; además, y con un accesorio digital se les cuantificaba la temperatura corporal y la saturación de oxígeno. Aquellos pacientes que cumplieron con la definición de caso sospechoso fueron enviados a su servicio médico correspondiente para la realización de RT-PCR en muestras de secreciones faríngeas de nariz y boca. Es importante mencionar que aproximadamente un $20 \%$ de los casos inciertos solamente refirieron un único síntoma menor y resultaron positivos para COVID-19, lo que subraya la presentación atípica de la infección en este conjunto de pacientes.

Con el propósito de asegurar la continuidad de las sesiones de hemodiálisis, se acondicionó un área aislada exclusiva para la atención de dichos pacientes. Las personas confirmadas de COVID-19 continuaron sus sesiones de hemodiálisis en unidades intrahospitalarias. Una vez que fueron dados de alta, se reincorporaron a los centros de diálisis ambulatorios del conjunto. Todos los pacientes convalecientes se mantuvieron aislados 21 días más y, al término de este periodo, se reintegraron a su máquina de hemodiálisis original.

Las unidades de hemodiálisis se apegaron a las recomendaciones de distanciamiento social, higiene de manos, estornudo de etiqueta y otras sugerencias publi- cadas por diversos autores de varios países, incluyendo México. ${ }^{11,12}$

De una población total de 3,723 pacientes en hemodiálisis crónica, $373(10 \%)$ se clasificaron como inciertos y de ellos un total de 160 pacientes resultaron ratificados con infección por SARS-CoV-2, que representó una incidencia acumulada de 430/10,000 pacientes. En los meses de mayo y junio se observó una tendencia mayor de casos sospechosos y confirmados, la cual se corresponde con la mayor incidencia de eventos que informó el Institute of Health Metrics Evaluation (IHME) para México en ese mismo periodo. ${ }^{13}$ Recientemente, Kunutsor $S$ y Laukkanen J publicaron ${ }^{14}$ una revisión sistemática y un metaanálisis de disfunción renal en COVID-19. Este informe incluyó 22 estudios de cohorte con 7,391 participantes, la incidencia combinada de COVID-19 durante el seguimiento de dos a 28 días en pacientes con enfermedad renal crónica preexistente (ERC) fue de 520/10,000 pacientes y para aquellos en hemodiálisis fue de 230/10,000.

En el presente trabajo, el promedio de edad fue similar a lo que reportan otros estudios: entre 57 y 66 años, lo cual reafirma que aquellos pacientes en hemodiálisis, infectados por SARS-CoV-2, frecuentemente son personas entre la sexta y séptima década de la vida. Más de la mitad de los individuos infectados eran hombres. Los síntomas más comunes en este estudio fueron: disnea, tos, fiebre y mialgias, lo cual es consistente con lo informado en la literatura internacional. $7,8,15,16$

La susceptibilidad de los pacientes con enfermedad renal crónica en hemodiálisis, para desarrollar infecciones de cualquier naturaleza, se debe principalmente a la uremia, la cual se asocia con alteraciones en los mecanismos de defensa primarios del huésped que, además, presenta disfunción de neutrófilos. Otras complicaciones que se 
ligan al cuadro urémico son: desnutrición, deficiencias vitamínicas, sobrecarga de hierro, alteraciones en el metabolismo de la glucosa, hiperparatiroidismo secundario, el procedimiento de hemodiálisis y la acumulación de toxinas urémicas.

También hay cambios en los procesos de la inmunidad mediada por células, en específico los linfocitos T; los cambios incluyen linfopenia, disminución de la proliferación de linfocitos in vitro, alteraciones en la inmunidad humoral con disminución de las concentraciones de inmunoglobulinas y limitación de la respuesta de los anticuerpos contra los antígenos. Desregulación en la síntesis de citocinas y daño en el receptor Fc de los macrófagos, sobre todo en pacientes urémicos. ${ }^{17,18}$
Otro factor que influye en la vulnerabilidad de estos pacientes es la incapacidad de aislamiento, ya que forzosamente tienen que trasladarse a su tratamiento, la mayoría de las veces en transporte público, hasta tres veces por semana. Las comorbilidades que tienen esos pacientes, como mal funcionamiento cardiovascular, diabetes mellitus, hipertensión arterial, obesidad y enfermedad pulmonar obstructiva crónica, son factores de riesgo que influyen en los malos resultados clínicos. La enfermedad cardiovascular isquémica fue más prevalente en el conjunto de pacientes que fallecieron, con un peligro relativo 31 veces mayor. ${ }^{19-21}$

Wu y colaboradores ${ }^{22}$ encontraron que, de sus 49 pacientes hospitalizados en hemodiálisis en Wuhan, China,

Tabla 2: Características clínicas de los pacientes con COVID-19 de acuerdo con su evolución.

\begin{tabular}{|c|c|c|c|c|}
\hline & $\begin{array}{c}\text { Total } \\
(\mathrm{N}=160)\end{array}$ & $\begin{array}{l}\text { Recuperados } \\
\qquad(\mathrm{N}=87)\end{array}$ & $\begin{array}{l}\text { Fallecidos } \\
(\mathrm{N}=73)\end{array}$ & \\
\hline Variables & $\mathrm{n}(\%)$ & $\mathrm{n}(\%)$ & $\mathrm{n}(\%)$ & $\mathrm{p}$ \\
\hline Edad** $^{\star *}$ & $55 \pm 15(20-88)$ & $54 \pm 15(24-82)$ & $57 \pm 14(20-88)$ & 0.238 \\
\hline \multicolumn{5}{|l|}{ Género } \\
\hline Femenino & $67(42)$ & $33(38)$ & $34(47)$ & 0.305 \\
\hline Masculino & $93(58)$ & $54(62)$ & $39(53)$ & \\
\hline \multicolumn{5}{|l|}{ Etiología } \\
\hline Nefropatía diabética & $64(40)$ & $31(36)$ & $33(45)$ & 0.610 \\
\hline Etiología no determinada & $46(29)$ & $29(33)$ & $17(20)$ & 0.350 \\
\hline Nefroangioesclerosis & $31(20)$ & $18(21)$ & $13(18)$ & 0.430 \\
\hline Otras & 19 (11) & $9(10)$ & $11(15)$ & 0.689 \\
\hline Índice de masa corporal $\left(\mathrm{kg} / \mathrm{m}^{2}\right)^{\star \star \star}$ & $26 \pm 5.3$ & $26 \pm 5.5$ & $26 \pm 5.1$ & 0.184 \\
\hline Tiempo en hemodiálisis (meses) & $49 \pm 38$ & $45 \pm 36$ & $55 \pm 39$ & 0.123 \\
\hline \multicolumn{5}{|l|}{ Grupo sanguíneo } \\
\hline $0+$ & $102(64)$ & $59(68)$ & $43(58)$ & 0.516 \\
\hline$A+$ & $35(22)$ & $18(21)$ & $17(23)$ & 0.314 \\
\hline$B+$ & $20(13)$ & $10(11)$ & $10(14)$ & 0.267 \\
\hline $\mathrm{AB}+$ & $3(1)$ & - & $3(4)$ & - \\
\hline \multicolumn{5}{|l|}{ Comorbilidades } \\
\hline Enfermedad cardiovascular & $46(29)$ & $19(22)$ & $27(37)$ & $0.049^{*}$ \\
\hline Diabetes mellitus & $64(40)$ & $35(40)$ & $29(40)$ & 0.350 \\
\hline Hipertensión arterial & $34(21)$ & $16(18)$ & $18(25)$ & 0.270 \\
\hline Enfermedad pulmonar obstructiva & $16(10)$ & 10 (11) & $6(8)$ & 0.410 \\
\hline Otras & $10(6)$ & $6(7)$ & $4(5)$ & 0.890 \\
\hline Hemoglobina $(\mathrm{g} / \mathrm{dL})^{\star \star \star}$ & $10 \pm 2.1$ & $9.9 \pm 2.1$ & $10 \pm 2.2$ & 0.627 \\
\hline Albúmina $(\mathrm{g} / \mathrm{dL})^{\star \star \star}$ & $3.5 \pm 0.43$ & $3.6 \pm 0.43$ & $3.5 \pm 0.43$ & 0.229 \\
\hline Linfocitos (núm/mL) & $1,066 \pm 375$ & $1,142 \pm 396$ & $978 \pm 330$ & $0.005^{\star}$ \\
\hline Peso normal & $60(40)$ & $39(45)$ & $20(27)$ & \\
\hline Sobrepeso & $100(60)$ & $48(55)$ & $54(73)$ & $0.022^{*}$ \\
\hline
\end{tabular}


Tabla 3: Factores de riesgo para COVID-19 en pacientes en hemodiálisis crónica.

\begin{tabular}{|c|c|c|}
\hline Variable & $\begin{array}{l}\text { Fallecidos } \\
\text { RR (IC 95\%) }\end{array}$ & $p^{*}$ \\
\hline \multicolumn{3}{|l|}{ Género } \\
\hline Masculino & $1.211(0.841-1.740)$ & ns \\
\hline Femenino & $0.871(0.667-1.137)$ & ns \\
\hline \multicolumn{3}{|l|}{ Edad (años) } \\
\hline$<55$ & 0.840 (0.591-1.193) & ns \\
\hline$>55$ & $1.150(0.872-1.516)$ & ns \\
\hline \multicolumn{3}{|l|}{ Comorbilidad } \\
\hline $\begin{array}{l}\text { Enfermedad } \\
\text { cardiovascular } \\
\text { isquémica }\end{array}$ & $1.31(1.16-1.45)$ & ns \\
\hline \multicolumn{3}{|l|}{ Peso } \\
\hline Normal & 1.32 (1.046-1.673) & ns \\
\hline Sobrepeso & $0.603(0.388-0.937)$ & $<0.05$ \\
\hline \multicolumn{3}{|l|}{ Linfocitos (mL) } \\
\hline$<1,000$ & 1.65 (1.292-2.199) & $<0.05$ \\
\hline$>1,000$ & $0.517(0.349-0.767)$ & ns \\
\hline $\begin{array}{l}\mathrm{RR}(\mathrm{IC} 95 \%)=\text { riesg } \\
{ }^{*} \mathrm{p} \leq 0.05=\text { estadísti } \\
\mathrm{ns}=\text { no significativo. }\end{array}$ & $\begin{array}{l}\text { o (intervalo de confianza } \\
\text { e significativo. }\end{array}$ & $\%)$. \\
\hline
\end{tabular}

47 de $90 \%$ tenían menos fiebre, 49 de $71 \%$ menos tos seca y más linfopenia; en comparación con el grupo con COVID-19 de la población general. Resultados muy parecidos a los de este estudio.

Estos hallazgos resaltan la importancia de presentaciones clínicas atípicas en pacientes en diálisis, como la ausencia de fiebre y tos, también encontrada en reportes previos. ${ }^{23-25}$

En relación al curso clínico de los pacientes, de los 160 contagios verificados se recuperaron 87 (55\%) y fallecieron 73 (45\%). Para una tasa de letalidad de $45 \%$, la cual es un poco elevada, comparada con otros informes publicados, que van desde $20-35 \% .4,26,27$ Pio-Abreu A y colegas $^{28}$ publicaron la serie de casos más grande compartida hasta ahora, con 37,852 pacientes en hemodiálisis y 1,291 pacientes confirmados con COVID-19, con una tasa de incidencia de $341 / 10,000$ pacientes, y una tasa de letalidad de $28 \%$. En esta serie en México, la incidencia fue de 430/10,000 y una letalidad de $45 \%$. Esta diferencia de mayor incidencia de infecciones y de mayor tasa de letalidad en los pacientes estudiados se explica por una proporción de casos no diagnosticados, lo que incrementa el índice de positividad, que actualmente es de casi $50 \%$.

En el análisis de los factores de riesgo que se identificaron para fallecer por COVID-19, los más salientes fueron la enfermedad cardiovascular isquémica, la edad mayor a
55 años, el número de linfocitos totales inferior a 1,000/ $\mathrm{mL}$ y el sobrepeso.

Finalmente, los pacientes con enfermedad renal crónica en hemodiálisis son un grupo de muy elevada susceptibilidad para la infección por SARS-CoV-2 por las características ya mencionadas y con riesgo alto de fallecer cuando se les compara con la población en general.

\section{REFERENCIAS}

1. Huang C, Wang Y, Li X, Ren L, Zhao J, Hu Y et al. Clinical features of patients infected with 2019 novel coronavirus in Wuhan, China. Lancet. 2020; 395 (10223): 497-506.

2. Guan WJ, Ni ZY, Hu Y, Liang WH, Ou CQ, He JX et al. Clinical characteristics of coronavirus disease 2019 in China. N Engl J Med. 2020; 382 (18): 1708-1720.

3. World Health Organization. Novel Coronavirus (2019-nCov) Situation Report-163, 2020. [Acceso 20 de julio de 2020] Available in: https://www.who.int/docs/default-source/coronaviruse/situationreports/20200701-covid-19-sitrep-163

4. Zhou F, Yu T, Du R, Fan G, Liu Y, Liu Z et al. Clinical course and risk factors for mortality of adult inpatients with COVID-19 in Wuhan, China: a retrospective cohort study. Lancet. 2020; 395 (10229): 1054-1062.

5. Yi Y, Lagniton PNP, Ye S, Li E, Xu RH. COVID-19: what has been learned and to be learned about the novel coronavirus disease. Int J Biol Sci. 2020; 16 (10): 1753-1766.

6. Madjid M, Safavi-Naeini P, Solomon SD, Vardeny O. Potential effects of coronaviruses on the cardiovascular system: a review. JAMA Cardiol. 2020; 5 (7): 831-840.

7. Zhu J, Ji P, Pang J, Zhong Z, Li H, He C et al. Clinical characteristics of 3062 COVID-19 patients: a meta-analysis. J Med Virol. 2020; 92 (10): 1902-1914. doi: 10.1002/jmv.25884.

8. Ma Y, Diao B, Lv X, Zhu J, Liang W, Liu L el al. 2019 novel coronavirus disease in hemodialysis (HD) patients: report from one HD center in Wuhan, China. medRxiv. 2020. doi: 10.1101/2020.02.24.20027201.

9. Valeri AM, Robbins-Juarez SY, Stevens JS, Ahn W, Rao MK, Radhakrishnan J et al. Presentation and outcomes of patients with ESKD and COVID-19. J Am Soc Nephrol. 2020; 31 (7): 1409-1415.

10. Aydin Bahat K, Parmaksiz E, Sert S. The clinical characteristics and course of COVID-19 in hemodialysis patients. Hemodial Int. 2020; 24 (4): 534-540. doi: 10.1111/hdi.12861.

11. Kliger AS, Cozzolino M, Jha V, Harbert G, Ikizler TA. Managing the COVID-19 pandemic: international comparisons in dialysis patients. Kidney Int. 2020; 98 (1): 12-16.

12. Vega-Vega O, Arvizu-Hernández M, Domínguez-Cherit JG, SierraMadero J, Correa-Rotter R. Prevención y control de la infección por coronavirus SARS-CoV-2 (COVID-19) en unidades de hemodiálisis. Salud Publica Mex. 2020; 62 (3): 341-347.

13. Institute Health of Metrics Evaluation. [Acceso en septiembre de 2020] Sitio web: https://covid19. healthdata.org/mexico?view=totaldeaths\&tab $=$ trend

14. Kunutsor SK, Laukkanen JA. Renal complications in COVID-19: a systematic review and meta-analysis. Ann Med. 2020; 52 (7): 345353. doi: 10.1080/07853890.2020.1790643.

15. Cheng Y, Luo R, Wang K, Zhang M, Wang Z, Dong L et al. Kidney disease is associated with in-hospital death of patients with COVID-19. Kidney Int. 2020; 97 (5): 829-838.

16. Xiong F, Tang H, Liu L, Tu C, Tian JB, Lei CT et al. Clinical Characteristics of and Medical Interventions for COVID-19 in Hemodialysis Patients in Wuhan, China. J Am Soc Nephrol. 2020; 31 (7): 1387-1397. 
17. Vaziri ND, Pahl MV, Crum A, Norris K. Effect of uremia on structure and function of immune system. J Ren Nutr. 2012; 22 (1): 149-156.

18. Betjes MG. Immune cell dysfunction and inflammation in end-stage renal disease. Nat Rev Nephrol. 2013; 9 (5): 255-265. doi: 10.1038/ nrneph.2013.44.

19. Kwan BC, Leung CB, Szeto CC, Wong VW, Cheng YL, Yu AW et al. Severe acute respiratory syndrome in dialysis patients. J Am SoC Nephrol. 2004; 15 (7): 1883-1888.

20. Naicker S, Yang CW, Hwang SJ, Liu BC, Chen JH, Jha V. The Novel Coronavirus 2019 epidemic and kidneys. Kidney Int. 2020; 97 (5): 824-828.

21. Goicoechea M, Sánchez Cámara LA, Macías N, Muñoz de Morales A, Rojas AG, Bascuñana A et al. COVID-19: clinical course and outcomes of 36 hemodialysis patients in Spain. Kidney Int. 2020; 98 (1): $27-34$.

22. Wu J, Li J, Zhu G, Zhang Y, Bi Z, Yu Y et al. Clinical features of maintenance hemodialysis patients with 2019 novel coronavirusinfected pneumonia in Wuhan, China. Clin J Am Soc Nephrol. 2020; 15 (8): 1139-1145.
23. Li J, Xu G. Lessons from the experience in Wuhan to reduce risk of COVID-19 infection in patients undergoing long-term hemodialysis. Clin J Am Soc Nephrol. 2020; 15 (5): 717-719.

24. Ferrey AJ, Choi G, Hanna RM, Chang Y, Tantisattamo E, Ivaturi K et al. A case of novel coronavirus disease 19 in a chronic hemodialysis patient presenting with gastroenteritis and developing severe pulmonary disease. Am J Nephrol. 2020; 51 (5): 337-342.

25. Alberici F, Delbarba E, Manenti C, Econimo L, Valerio F, Pola A et al. A report from the Brescia Renal COVID Task Force on the clinical characteristics and short-term outcome of hemodialysis patients with SARS-CoV-2 infection. Kidney Int. 2020; 98 (1): 20-26.

26. Henry BM, Lippi G. Chronic kidney disease is associated with severe coronavirus disease 2019 (COVID-19) infection. Int Urol Nephrol. 2020; 52 (6): 1193-1194.

27. Zheng YY, Ma YT, Zhang JY, Xie X. COVID-19 and the cardiovascular system. Nat Rev Cardiol. 2020; 17 (5): 259-260.

28. Pio-Abreu A, do Nascimento MM, Vieira MA, de Menezes Neves PDM, Lugon JR, Sesso R. High mortality of CKD patients on hemodialysis with Covid-19 in Brazil. J Nephrol. 2020; 33 (5): 875877. doi: 10.1007/s40620-020-00823-z. 